\title{
Multifractal Analysis of Heart Rate Variability by Applying Wavelet Transform Modulas Maxima Method
}

\author{
Evgeniya Gospodinova, Galya Georgieva-Tsaneva, Penio Lebamovski \\ Institute of Robotics, Bulgarian Academy of Sciences, Sofia, Bulgaria
}

\begin{abstract}
The analysis of heart rate variability is based on the intervals between the successive heartbeats and thanks to it information about the functional state of the person can be obtained and the dynamics of its change can be traced. The nonlinear dynamics methods provide additional, prognostic information about the patient's health, complementing traditional analyses and are considered potentially promising tools for assessing heart rate variability. In this article, studies have been carried out to identify the mono- and multifractal properties of two groups of people: healthy controls and patients with arrhythmia using Wavelet Transform Modulas Maxima Method. The obtained results from the studies show that for healthy subjects the multifractal spectrum is broader than the spectrum of patients with arrhythmia. The value of the Hurst exponent is lower in healthy controls, and in patients with arrhythmia this parameter tends to one. For the healthy subjects, the scaling exponent showed nonlinear behaviour, while for patients with arrhythmia it was linear. This indicates that heart rate variability in healthy controls has multifractal behaviour while patients with arrhythmia have monofractal behaviour. The finding may be useful in diagnosing subjects with cardiovascular disease, as well as in predicting future diseases, as the heart rate variability changes at the slightest deviation in the health status of subjects before the onset of relevant signs of the disease.
\end{abstract}

Keywords-RR time series; heart rate variability; wavelet transform modulas maxima method; monofractal; multifractal

\section{INTRODUCTION}

The effectiveness of the modern medical technologies is closely linked to the improvement of the methods and the instruments for monitoring and analysing the condition of patients during their treatment. In medicine the problem with patients' clinical surveillance occupies a special place, as the monitoring of their current state can be of vital importance.

The use of electrocardiographic data for analysis of the cardiac activity of the patient is a generally accepted method. The presentation of heart rhythm as a dynamic row of RR time intervals (the distances between the R-tops of the electrocardiogram) and the mathematical analysis of this data [1] is widely used in the research of the cardiac activity. Based on the RR interval series, heart rate variability (HRV) is determined, which is one of the most accessible physiological parameters [2] reflecting the processes of autonomic regulation in the cardiovascular system. The dynamic characteristics of the heart rate make it possible to assess the severity of changes in the sympathetic and parasympathetic activity of the autonomic nervous system in changing the patient's health. The sympathetic branch reduces the intervals between heartbeats [3], while the parasympathetic branch increases them.

In modern cardiology, more and more attention is paid to the analysis of heart rate variability and more specifically to the changes in heart rate intervals [4]. Heart rate is the most objective characteristic of the functional state of the human body and depends on several factors: age, gender, environmental conditions, stress, body temperature, etc. [5, 6]. HRV analysis is a unique diagnostic technique that allows not only to assess the functional state of the human body, but also to monitor its dynamics and to identify the occurrence of pathological conditions when they are at a very early stage. The heavy physical work, the psychological stress, as well as the disease states of the human body lead to an increase in heart rate and to a decrease in HRV. Conversely, when the body is at rest, the heart rate is usually lower and the HRV is higher.

The mathematical methods for assessing the functional state of the human body through the study of HRV are combined into the following two groups: linear and nonlinear methods.

The linear methods include time-domain analysis and frequency-domain analysis. These methods are standardized, knowing the reference values of the studied parameters, but this is often not enough to characterize the complex dynamics of the RR time series of heart rate.

The nonlinear methods such as: Poincare plot, Detrended Fluctuation Analysis (DFA), Multifractal Detrended Fluctuation Analysis (MFDFA), Wavelet Transform Modulas Maxima Method (WTMM), AppEn, SampEn [7, 8, 9, 10] and others are not standardized, which is the reason for their limited use in clinical practice. The nonlinear methods for HRV analysis are based on the theory of chaos and fractals. These methods are in the process of active research, and it is expected that in the near future they will be able to give a new idea of the dynamics of heart rate in the context of physiological changes in patients with cardiovascular disease. Practically, each cardio interval contains elements of nonstationarity (fractal components) and for their evaluation in recent years methods of nonlinear dynamics are actively applied. These methods provide additional prognostic information of the studied signals, which complements the traditional analyses in the time and frequency domains.

This article is funded by the National Science Fund of Bulgaria (BNSF). 
To be able to deal with the problem of the accurate HRV assessment of the studied cardiac signals, it is necessary to choose an appropriate method of analysis that represents the dynamics of the heart rate.

The aim of this article is to analyse and evaluate HRV in two groups of people: healthy controls and patients with arrhythmia, using the Wavelet Transform Modulas Maxima method. The effectiveness of the method used was evaluated by statistical t-analysis.

The rest of the paper is organized as follows: Section II provides an overview of related research in the scientific literature. Section III focuses on the Wavelet Transform Modulas Maxima Method used in this paper for HRV analysis. Section IV describes the data used for the analyses performed. Section $\mathrm{V}$ presents the results and discussions. The final section (Section VI) of the article contains the conclusion that can be made from the obtained results.

\section{RELATED WORK}

In recent years, there has been an active introduction of the mathematical methods of analysis in the medical practice. Many scientists have studied the complex nature of the changes in the parameters of electrocardiographic (ECG) signals using nonlinear dynamic methods. The fractal and multifractal approach in the analysis of the cardiological data allows to obtain new knowledge and assessments that give an idea of the nonlinear dynamic processes taking place in them. As a result of the work of [11] on the heartbeat dynamics, the multifractal analysis has become a widely used tool for applied research, in cases where the non-stationary processes are limited by the application of the classical methods of analysis.

The multifractal analysis expands the possibilities for cardio-diagnostics based on the wavelet theory. The proposed approach by [12] for multiresolution wavelet analysis of heartbeat intervals allows distinguishing healthy patients from those with cardiac pathology. This universal approach is applicable in the analysis of non-stationary processes in the physical and biological sciences, including the analysis of ECG signals.

The authors in [13] proposed a technique for multifractal analysis to determine the degree of multifractality of the heart rate of patients suffering from partial seizures. The results show that the degree of multifractality varies depending on the severity of the disease.

The authors of [14] advise physicians to interpret the results obtained from fractal and traditional methods with caution, as they are still in the process of research and the measurements obtained are not fully described as biomarkers for clinical use.

In [15], the authors show how the entropy and the multifractal analysis can depict the dynamics of heart rate when students performed selective inhibition tasks. The results show that the entropy and the fractal markers outperform markers in the time and frequency range of the heart rate variability in distinguishing the cognitive tasks.
The WTMM method presented by Arneodo et al. [16] can be used to study the structures of inhomogeneous processes of various natures, based on wavelet and multifractal analysis.

Recent studies $[17,18]$ have shown that HRV changes in individuals infected with Covid-19 even before the onset of symptoms of the disease. This indicator can be useful for early detection of this disease.

The need to study HRV through the application of mathematical methods is determined by the fact that it accurately reflects the state of regulatory processes in the human body and provides information that is important in the diagnosis, prognosis, treatment, and prevention of diseases of various kinds.

\section{Methodology}

The choice of an appropriate mathematical method for time series analysis is determined by its flexibility and ability to be effectively applied to real processes. Among such universal methods for time series analysis, the WTMM method, which is discussed in this article, can be applied.

The WTMM method [19, 20, 21] is based on the mathematical tools of wavelet, fractal and multifractal analysis, which can reveal the hidden dynamics of the studied time series of heart rate in the context of mono- and multifractality.

The wavelet theory allows the studied signal to be analysed in more detail than Fourier analysis [22]. The wavelets are localized at both frequency and time, while the standard Fourier transform has only frequency localization.

The continuous wavelet transform (CWT) is used to decompose the continuous wavelet function. Unlike the Fourier transform, CWT provides the ability to build a time-frequency representation of the signal [23, 24], which achieves very good localization in time and frequency.

The dynamic characteristics of the RR interval series have fractal and in some cases multifractal properties [25, 26]. The fractal concept is related to processes (objects) that meet the following two criteria:

- Self-similarity: The process consists of many segments that are similar to each other and to the whole object.

- Fractional dimension: According to this criterion the fractal objects are distinguished from Euclidean ones, which are characterized by a dimension that is an integer.

The fractal processes are of two types: monofractal and multifractal [25]. The monofractal process is homogeneous in the sense that it has the same scaling properties, which both locally and globally can be characterized by a single scale indicator, such as: fractal dimension and Hurst exponent. Unlike the monofractal processes, the multifractal processes decompose into a large number of homogeneous fractal subsets, whose properties can be characterized by a spectrum of local fractal dimensions or Hearst exponents. 
The behaviour of the studied signal by applying the WTMM method is performed in two stages [27, 28, 29, 30]. In the first stage, a continuous wavelet transform is performed according to the following formula:

$W(a, b)=\frac{1}{a} \int_{0}^{T} x(t) \psi^{*}\left(\frac{t-b}{a}\right) d$

Where:

- $\mathrm{W}$ are the wavelet coefficients;

- $\quad$ a is a scale parameter, $a \in R^{+}$;

- $\mathrm{b}$ is the translation coefficient, $b \in R$;

- $\mathrm{x}(\mathrm{t})$ is the input signal;

- $\psi^{*}$ is a continuous function in the field of time and frequency, called the mother wavelet, which is complexly conjugated. The main purpose of the mother wavelet is to provide a function to generate daughter wavelets;

- $\mathrm{T}$ is the maximum value of time.

The scale parameter a can stretch or contract the signal under study. When the value of this parameter is small, the signal is compressed, which in turn leads to a more detailed graph. On the other hand, when the scale parameter is higher, the signal is stretched, which means that the resulting graph will be presented in lower detail.

To determine the singularity of the function, it is sufficient to use only the information about the maximum of the obtained wavelet coefficients, constructing the skeleton of the wavelet transform [31,32]. Following the lines of the skeleton, the behaviour of the singularities of the function $\mathrm{x}(\mathrm{t})$ can be traced [33].

The second stage of the WTMM method consists in the creation of partition functions $\mathrm{Z}(\mathrm{q}, \mathrm{a})$, which allow to obtain reliable estimates of the characteristics of the studied process:

$Z(q, a)=\sum_{l \in L(a)}\left(\sup _{a^{\prime} \leq a}\left|W\left(a^{\prime}, x_{l}\left(a^{\prime}\right)\right)\right|\right)^{q}$

Where:

- $\quad \mathrm{L}(\mathrm{a})$ is a set of all lines;

- 1 are the local maxima of the modules of the wavelet coefficients that exist for the scale a.

Equation (2) shows that the maximum value of the modulus is selected for each line at scales smaller than a set value of a. As a rule, it is expected that at small values of a, the partition function will have a power dependence, which will quantitatively characterize the scaling exponents $\tau(q)$ :

$Z(q, a) \sim a^{\tau(q)}$

The scaling exponent $\tau(q)$ is defined by the following expression:

$\tau(q) \sim \log _{10} Z(q, a) / \log _{10} a$

By selecting different values of the parameter q, a linear or nonlinear dependence of $\tau(q)$ can be obtained, depending on the type of the studied process [34]:
- if the process is monofractal, then the function $\tau(q)$ is linear and the exponent $h(q)=d \tau(q) / d q=$ const;

- if the process is multifractal, then the function $\tau(q)$ is nonlinear and the exponent $h(q)=d \tau(q) / d q \neq$ const.

By analogy with thermodynamic formalism, the spectrum of singularities is calculated on the basis of the Legender transformation:

$D(h)=q h(q)-\tau(q)$

The spectrum D (h) is determined by the set of values of the fractal dimensions of the original time series. The maximum of the spectral curve corresponds to the Hurst parameter. The following conclusions about the signal behaviour can be made from the value of the Hurst parameter:

- if $0<\mathrm{h}<0.5$, then the signal has anticorrelation dynamics;

- if $0.5<\mathrm{h}<1.0$, then the signal has a correlation behavior;

- if $\mathrm{h}=0.5$, it lacks correlation in the signal.

\section{DATA}

The application of wavelet and fractal analysis of the studied RR interval series is designed to determine and visually assess the degree of harmonization of the studied time series that have fractal-like structures. The purpose of this analysis is to identify functional and pathological changes, as well as to predict changes in the health status of patients.

To test whether HRV can provide information outside of linear indices, the following two groups of people were examined in this study:

- healthy controls (10 men and 10 women aged $56 \pm 4$ ).

- patients with arrhythmia (10 men and 10 women aged $58 \pm 3$ years).

\section{RESUltS AND DISCUSSION}

The software for the analysis of the fractal and multifractal properties of the test signals was created with MATLAB.

On Fig. 1A and Fig. 1B are shown RR interval series corresponding to the heart rate variations in a healthy individual and a sick patient with arrhythmia. Variations in a healthy individual are greater than those of a sick patient. This property can be used as a criterion in cardiovascular diagnosis. In practice, heart rate dynamics can be investigated using the linear methods by applying methods in the time- and frequency domains. The obvious shortcomings of these methods are that they can only be applied only to stationary time series and the heart rate shows heterogeneity and non-stationary of its fluctuation.

On Fig. 1C and Fig. 1D are shown the results of the wavelet transform and the graphics obtained have tree structures. The tree structure is more pronounced in the healthy patient. This property allows revealing the structure of the RR interval series and can also be used as a diagnostic criterion. The colour code of the graphics presents the values of wavelet 
coefficients. The light colours correspond to the higher absolute values of the coefficients and darker colours correspond to the lower values.

Important information about the behaviour of the studied $\mathrm{RR}$ interval series is also contained in the wavelet skeleton of the local maxima lines on each scale of wavelet coefficients matrix (Fig. 1E and Fig. 1F). The local maximum modules of wavelet transformation $|\mathrm{W}(\mathrm{a}, \mathrm{B})|$ there are the greatest values in those points of the analysed function in which it undergoes the most significant changes (jumps).

On Fig. 2A and Fig. 2B are shown the partition functions $\mathrm{Z}(\mathrm{q}, \mathrm{a})$ for a healthy subject and for a patient with arrhythmia. The calculation of the $\mathrm{Z}(\mathrm{q}, \mathrm{a})$ allow the signal fluctuations to be monitored. Positive values of the parameter $q$ accentuate the large fluctuations of the signal (strong inhomogeneity), while the negative values of $q$ accentuate on small fluctuations.
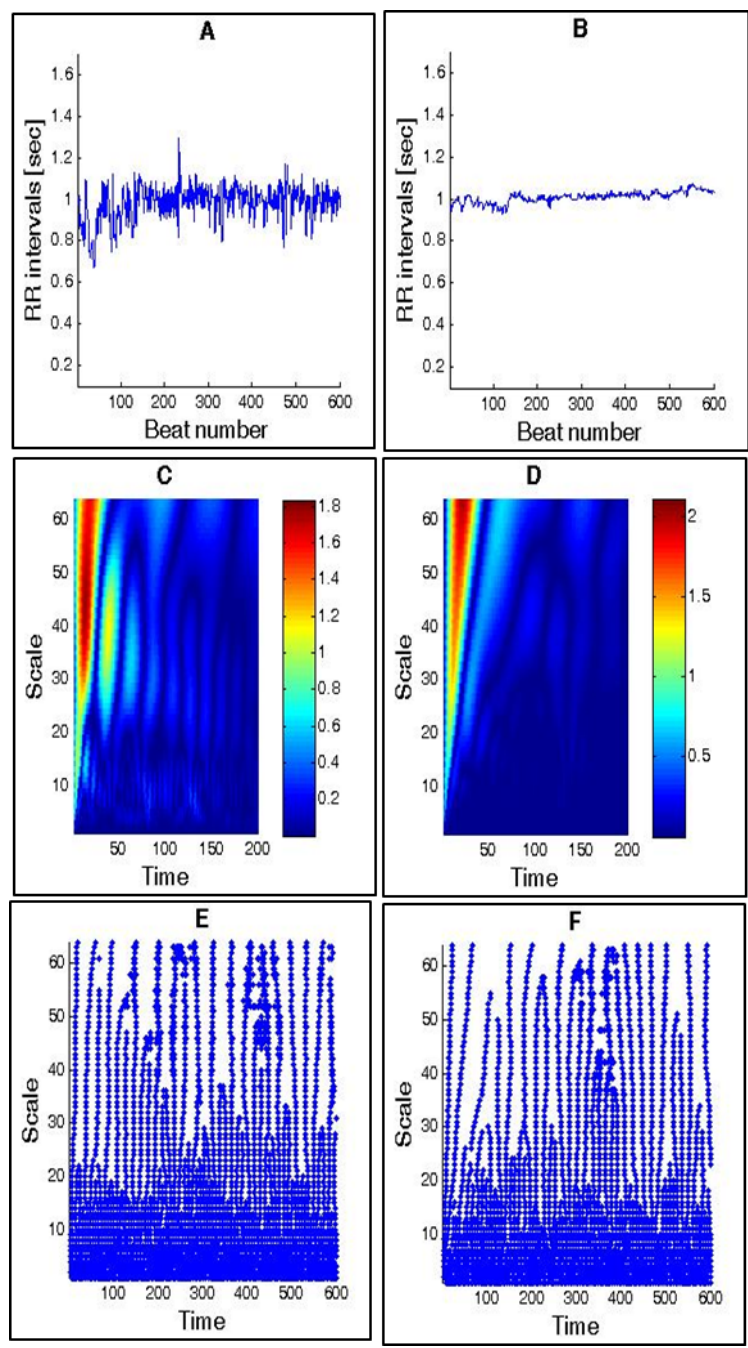

Fig. 1. RR Time Series for Healthy Subject (A) and for Subject with Arrhythmia (B); CWT Coefficients Plot in the Case of RR Intervals for Healthy (C) and Subject with Arrhythmia (D); WTMM Skeleton Plots for Healthy Subject (E) and Subject with Arrhythmia (F).
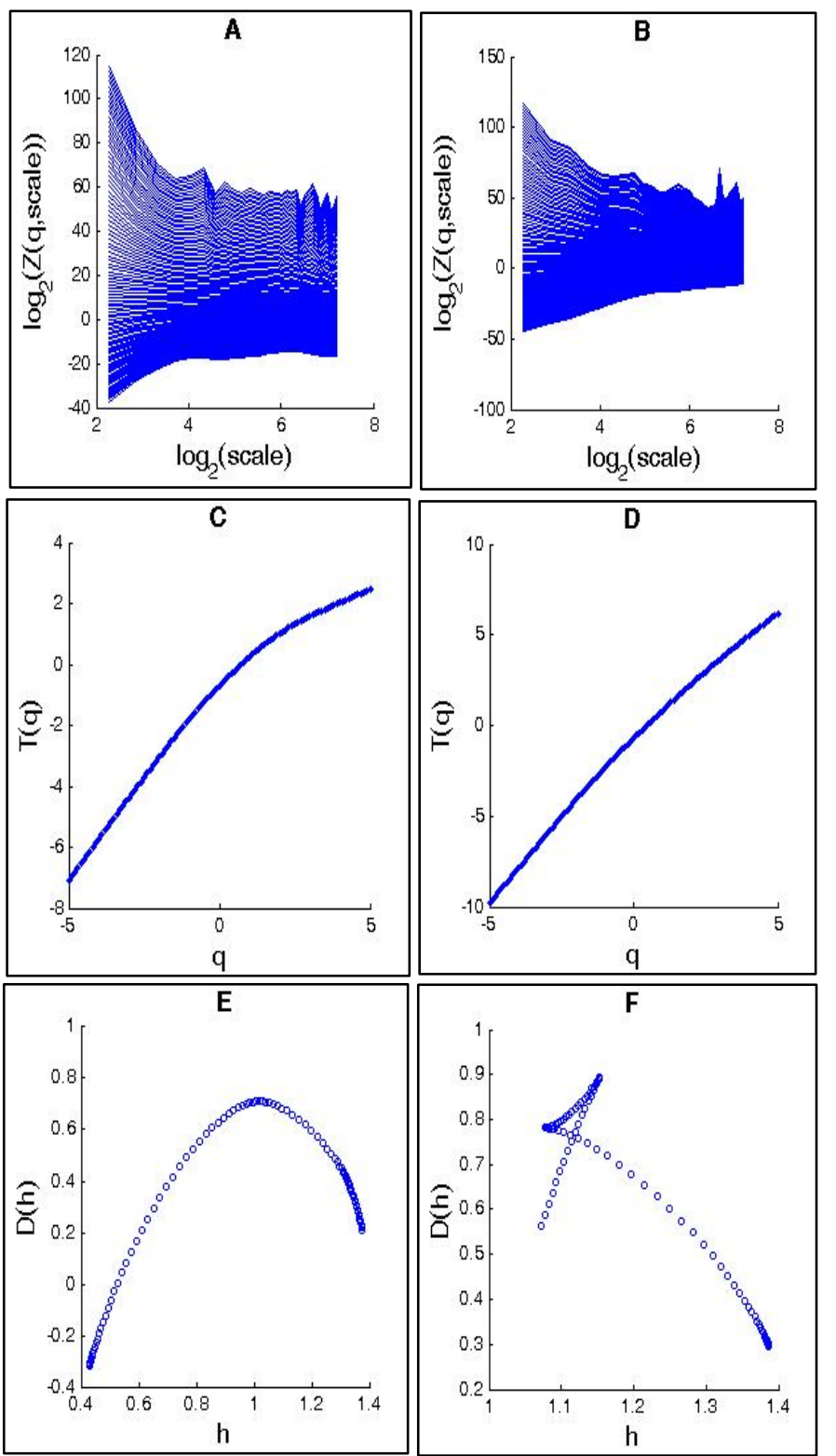

Fig. 2. Partition Functions for Healthy Subject (A) and for Subject with Arrhythmia (B); Scaling Exponent $\tau(\mathrm{q})$ for Healthy Subject (C) and for Subject with Arrhythmia (D); Multifractal Spectrum for for Healthy Subject (E) and for Subject with Arrhythmia (F).

The function $\tau(q)$ characterizes the fractal properties of the $\mathrm{RR}$ time series investigated. The graphics of the scaling exponent $\tau(\mathrm{q})$ have been shown on Fig. 2C and Fig. 2D for a healthy subject and for a patient with arrhythmia. For the healthy subject, the $\tau(q)$ spectrum has nonlinear behaviour and for the patient with arrhythmia this function is linear.

The statistical differences for multifractal spectrum between the RR time series of the two investigated subjects have been shown on Fig. 2E and Fig. 2F. The key characteristics are: the width of the spectrum of the singularity and the value of the exponent corresponding to the maximum value of the spectrum (Hurst exponent). For healthy people, the singularity spectrum is wide with non-zero singularities. On the other hand, for the patient with arrhythmia the singularity 
spectrum is a very narrow range. In Table I is shown the values of the multifractal spectrum parameters: $\mathrm{h}_{\max }, \mathrm{h}_{\min }, \Delta \mathrm{h}=\mathrm{h}_{\max }{ }^{-}$ $\mathrm{h}_{\min }$ and the value of the Hurst exponent observed for healthy subjects and patients with arrhythmia. The results show that the width of the multifractal spectrum of the group: healthy controls are greater than the group: patients with arrhythmia. The value of the Hurst exponent is lower in healthy subjects. The studied parameters determined by t-test have statistical significance ( $\mathrm{p}$-value $<0.05$ ), therefore with these parameters the two studied groups can be distinguished.

The obtained results show that the heart rate of the healthy people is characterized by uneven dynamics in the form of multifractal behaviour, which cannot be detected by traditional methods, but can be observed using the WTMM method. In the pathological cases, the uneven dynamics of HRV is destroyed, which reflects the state of the cardiovascular system.

The results demonstrate the effectiveness of applying the WTMM method for HRV analysis as an additional measure that can expand and improve the information obtained from the $\mathrm{RR}$ time series in the diagnosis and prognosis of cardiovascular disease.

TABLE I. MUlTIFRACTAL SPECTRUM PARAMETERS AND HURST EXPONENT

\begin{tabular}{|c|c|c|c|}
\hline \multirow[t]{2}{*}{ Parameter } & $\begin{array}{l}\text { healthy controls } \\
\mathrm{N}=\mathbf{2 0}\end{array}$ & $\begin{array}{l}\text { patients -arrhythmia } \\
\mathrm{N}=20\end{array}$ & \multirow{2}{*}{ p-value } \\
\hline & mean \pm std & mean \pm std & \\
\hline $\mathrm{h}_{\max }$ & $1.42 \pm 0.12$ & $1.31 \pm 0.15$ & 0.01 \\
\hline $\mathrm{h}_{\min }$ & $0.30 \pm 0.20$ & $0.8 \pm 0.14$ & 0.0001 \\
\hline$\Delta \mathrm{h}=\mathrm{h}_{\max } \mathrm{h}_{\min }$ & $1.0 \pm 0.22$ & $0.6 \pm 0.20$ & 0.0001 \\
\hline Hurst & $0.72 \pm 0.10$ & $0.93 \pm 0.3$ & 0.01 \\
\hline
\end{tabular}

\section{CONCLUSION}

The results obtained in this article confirm the hypothesis that monofractality is a marker of pathological dynamics of heart rhythm in the case of cardiovascular disease such as arrhythmia. Conversely, it has been shown that multifractality is an indicator of a healthy individual. Therefore, HRV analysis using the WTMM method can be a useful approach to distinguish healthy controls from patients with arrhythmia, as the parameters studied have statistical significance (pvalue $<0.05$ ). Interpretation of the results of this type of analysis may be useful before the possibility of using this method for physiological or clinical studies.

\section{ACKNOWLEDGMENT}

This research work was carried out as part of the scientific project "Investigation of the application of new mathematical methods for the analysis of cardiac data" No KP-06-N22/5, date 07.12.2018, funded by the National Science Fund of Bulgaria (BNSF).

\section{REFERENCES}

[1] F. Shaffer, J. P. Ginsberg, "An Overview of Heart Rate Variability Metrics and Norms," Front Public Health, 5:258. doi: 10.3389/fpubh.2017.00258. PMID: 29034226; PMCID: PMC5624990, September 2017.

[2] F. Sessa, V. Anna, G. Messina, G. Cibelli, V. Monda, G. Marsala, M. Ruberto, A. Biondi, O. Cascio, G. Bertozzi, D. Pisanelli, F. Maglietta,
A. Messina, M. P. Mollica, M. Salerno, "Heart rate variability as predictive factor for sudden cardiac death,” Aging, 10(2), pp. 166-177, 2018. https://doi.org/10.18632/aging.101386.

[3] B. Grässler, B. Thielmann, I. Böckelmann, A. Hökelmann, "Effects of Different Training Interventions on Heart Rate Variability and Cardiovascular Health and Risk Factors in Young and Middle-Aged Adults: A Systematic Review", Frontiers in Physiology, vol.12, pages=532, 2021 https://www.frontiersin.org/article/10.3389/fphys. 2021.657274.

[4] S. U. Marasingha-Arachchige, J. Á. Rubio-Arias, P. E. Alcaraz, L. H. Chung, "Factors that affect heart rate variability following acute resistance exercise: A systematic review and meta-analysis,” Journal of Sport and Health Science, 2020, ISSN 2095-2546. https://doi.org/10.1016/j.jshs.2020.11.008.

[5] F. Shaffer, J. P. Ginsberg, "An Overview of Heart Rate Variability Metrics and Norms," Frontiers in public health, vol. 5, pages $=258$, 2017. https://doi.org/10.3389/fpubh.2017.00258.

[6] R. McCraty, F. Shaffer, "Heart rate variability: new perspectives on physiological mechanisms, assessment of self-regulatory capacity, and health risk," Glob Adv Health Med, vol.4, issue 1, pp. 46-61, 2015. https://doi.org/10.7453/gahmj.2014.073.

[7] Z. Germán-Salló, M. Germán-Salló, "Non-linear Methods in HRV Analysis”, Procedia Technology, Vol. 22, pp. 645-651, 2016, ISSN 2212-0173, https://doi.org/10.1016/j.protcy.2016.01.134.

[8] C. Fiskum, T. G. Andersen, X. Bornas, P. M. Aslaksen, M. A. Flaten, K. Jacobsen, "Non-linear Heart Rate Variability as a Discriminator of Internalizing Psychopathology and Negative Affect in Children With Internalizing Problems and Healthy Controls," Frontiers in Physiology, vol. 9, pp.561, 2018. https://www.frontiersin.org/article/10.3389/fphys. 2018.00561.

[9] P.Melillo, M. Bracale, L. Pecchia, "Nonlinear Heart Rate Variability features for real-life stress detection. Case study: students under stress due to university examination,” BioMed Eng OnLine, vol 10(96), 2011. https://doi.org/10.1186/1475-925X-10-96.

[10] T. Henriques, M. Ribeiro, A. Teixeira, L. Castro, L. Antunes, C. CostaSantos, "Nonlinear Methods Most Applied to Heart-Rate Time Series: A Review," Entropy (Basel, Switzerland), 22(3), 309, 2020. https://doi.org/10.3390/e22030309.

[11] P. Ivanov, L. Amaral, A. Goldberger, S. Havlin, M. G. Rosenblum, Z. R. Struzik, H. E. Stanley, "Multifractality in human heartbeat dynamics". Nature 399, 461-465, 1999. https://doi.org/10.1038/20924.

[12] S. Thurner, M. C. Feurstein, M. C. Teich, „Multiresolution Wavelet Analysis of Heartbeat Intervals Discriminates Healthy Patients from Those with Cardiac Pathology“. Phys. Rev. Lett. 80, 1544, 1998.

[13] D. Ghosh, S. Dutta, S. Chakraborty, S. Samanta, "Epileptic Seizure: A New Approach for Quantification of Autonomic Deregulation with Chaos Based Technique”. Transl Biomed. 2017, 8:1. https://doi.org/10.21767/2172-0479.100106.

[14] J. Sen, D. McGill. "Fractal analysis of heart rate variability as a predictor of mortality: A systematic review and meta-analysis". An Interdisciplinary Journal of Nonlinear Science, Volume 28, Issue 7, 2018. https://doi.org/10.1063/1.5038818.

[15] P. Bouny, L. M. Arsac, C. R. Touré, V. Deschodt-Arsac, „Entropy and Multifractal-Multiscale Indices of Heart Rate Time Series to Evaluate Intricate Cognitive-Autonomic Interactions“. Entropy. 2021; 23(6):663. https://doi.org/10.3390/e23060663.

[16] A. Arneodo, B. Audit, E. Bacry, S. Manneville, J.F. Muzy, S.G. Roux, "Thermodynamics of fractal signals based on wavelet analysis: Application to fully developed turbulence data and DNA sequences". Physica A-statistical Mechanics and Its Applications - PHYSICA A. 254. 24-45, 1998. https://doi.org/10.1016/S0378-4371(98)00002-8.

[17] MBA Mol, MTA Strous, FHM van Osch, FJ Vogelaar, DG Barten, M. Farchi, NA Foudraine, Y. Gidron, "Heart-rate-variability (HRV), predicts outcomes in COVID-19,” PLoS One., vol 16 (10), October 2021, doi: 10.1371/journal.pone.0258841. PMID: 34710127; PMCID: PMC8553073.

[18] S.-A. Ouadfeul, "Multifractal behavior of SARS-CoV2 COVID-19 pandemic spread, case of: Algeria, Russia, USA and Italy”, medRxiv, 2020. https://doi.org/10.1101/2020.09.16.20196188. 
[19] H. Salat, R. Murcio, E. Arcaute, "Multifractal methodology", Physica A: Statistical Mechanics and its Applications, vol. 473, pp. 467-487, 2017, ISSN 0378-4371, https://doi.org/10.1016/j.physa.2017.01.041.

[20] A. Arneodo, B. Audit, P. Kestener, S. Roux, "Wavelet-based multifractal analysis”. Scholarpedia. 3. 4103, 2008. https://doi.org/10.4249/scholarpedia.4103.

[21] P. Ivanov, A. Luis, A. L. Goldberger, H. Shalomo, H. E. Stanley, Z. Struzik, "From $1 / \mathrm{f}$ noise to multifractal cascades in heartbeat dynamics," Chaos, vol 11(3), pp. 641-645q 2001. https://doi.org/10.1063/1. 1395631.

[22] G. Dodin, P. Vandergheynst, P. Levoir, C. Cordier, L. Marcourt, "Fourier and wavelet transform analysis, a tool for visualising regular patterns in dna," Journal of Theoretical Biology, 206(ARTICLE), 2000, pp.323-326.

[23] V. I. Kovalchuk, O. S. Svechnikova, L. A. Bulavin, "Multifractal Analysis of Cardiac Series and Predictors of Sudden Cardiac Death," Ukrainian Journal of Physics, vol. 66, No.10, 879, 2021. https://doi.org/10.15407/ujpe66.10.879.

[24] D. Makowiec, R. Galaska, A. Rynkiewicz, J. Wdowczyk-Szulc, "Multifractal estimators of short-time autonomiccontrol of the heart rate”, Proceedings of the International Multiconference on Computer Science and Information Technology, vol. 4, pp. 405-411, 2009.

[25] H. E. Stanley, L. A. Amaral, A. L. Goldberger, S. Havlin, P. Ivanov, and C. K. Peng, "Statistical physics and physiology: monofractal and multifractal approaches," Physica A, vol. 270, , pp. 309-324, 1999.

[26] E. Gerasimova, B. Audit, S. G. Roux, A. Khalil, O. Gileva, F. Argoul, O. Naimark, A. Arneodo, "Wavelet-based multifractal analysis of dynamic infrared thermograms to assist in early breast cancer diagnosis," Frontiers in physiology, vol. 5, 176, 2014. https://doi.org/10.3389/fphys.2014.00176.

[27] A. Puckovs, A. Matvejevs, "Wavelet Transform Modulus Maxima Approach for World Stock Index Multifractal Analysis,” Information
Technology and Management Science, December 2012, https://doi.org/10.2478/v10313-012-0016-5.

[28] A. Arneodo, B. Audit, N. Decoster, J. F. Muzy, C. Vaillant, “A wavelet based multifractal formalism: application to DNA sequences, satellite images of the cloud structure and stock market data," in The Science of Disasters: Climate Disruptions, Heart Attacks, and Market Crashes, eds A. Bunde, J. Kropp, and H. J. Schellnhuber (Berlin: Springer Verlag), pp. 26-102, 2002.

[29] J. F. Muzy, E. Bacry, A. Arneodo, "Wavelets and multifractal formalism for singular signals: application to turbulence data," Phys. Rev. Lett. Vol. 67, Issue 25, pp. 3515-3518, 1991. https://doi.org/10.1103/PhysRevLett.67.3515.

[30] J.-F. Muzy, E. Bacry, A. Arneodo, "Multifractal formalism for fractal signals: The structure-function approach versus the wavelet-transform modulus-maxima method,” Phys. Rev. E , vol. 47, issue 2, pp. 875-884, 1993. https://doi.org/10.1103/PhysRevE.47.875.

[31] J.-F. Muzy, E. Bacry, A. Arneodo, "The multifractal formalism revisited with wavelets,” Int. J. Bifurc. Chaos, vol. 4, pp. 245-302, 1994.

[32] R. Galaska, D. Makowiec, A. Dudkowska, A. Koprowski, K. Chlebus, J. Wdowczyk-Szulc, A. Rynkiewicz, "Comparison of wavelet transform modulus maxima and multifractal detrended fluctuation analysis of heart rate in patients with systolic dysfunction of left ventricle," Ann Noninvasive Electrocardiol, vol. 13(2), pp. 155-64, 2008. doi: 10.1111/j.1542-474X.2008.00215.x. PMID: 18426441; PMCID: PMC6932668.

[33] P. S. Addison, "The illustrated wavelet transform handbook: introductory theory and applications in science, engineering, medicine and finance”. CRC press; January 2017.

[34] P. Lio, "Wavelets in bioinformatics and computational biology: state of art and perspectives," Bioinformatics, vol 19(1), pp.2-9, 2003. 